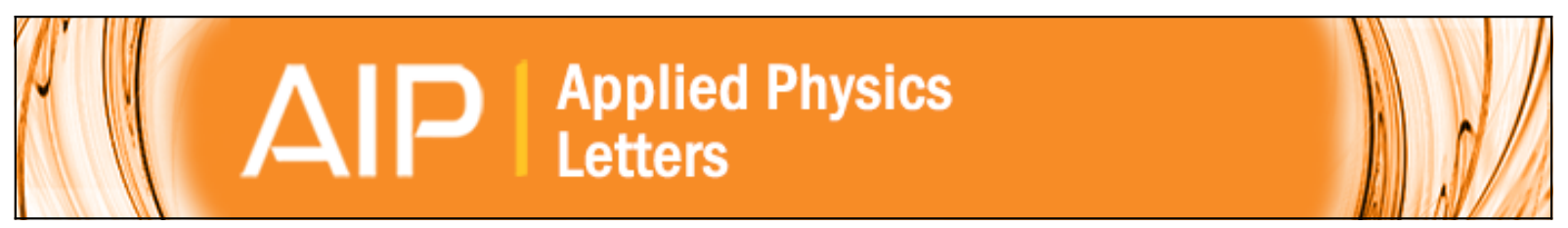

\title{
Stable planar single-layer hexagonal silicene under tensile strain and its anomalous Poisson's ratio
}

Baolin Wang, Jiangtao Wu, Xiaokun Gu, Hanqing Yin, Yujie Wei, Ronggui Yang, and Mildred Dresselhaus

Citation: Applied Physics Letters 104, 081902 (2014); doi: 10.1063/1.4866415

View online: http://dx.doi.org/10.1063/1.4866415

View Table of Contents: http://scitation.aip.org/content/aip/journal/apl/104/8?ver=pdfcov

Published by the AIP Publishing

\section{Articles you may be interested in}

Internal-strain effect on the valence band of strained silicon and its correlation with the bond angles

J. Appl. Phys. 115, 063702 (2014); 10.1063/1.4864217

Origin of anomalous strain effects on the molecular adsorption on boron-doped graphene

J. Chem. Phys. 139, 044709 (2013); 10.1063/1.4816365

Quantum mechanics based force field for carbon (QMFF-Cx) validated to reproduce the mechanical and thermodynamics properties of graphite

J. Chem. Phys. 133, 134114 (2010); 10.1063/1.3456543

Carbon nanotube films change Poisson's ratios from negative to positive

Appl. Phys. Lett. 97, 061909 (2010); 10.1063/1.3479393

Computational Protocols for Viscoelastic Material Property Characterizations without the Use of Poisson's Ratios AIP Conf. Proc. 1255, 37 (2010); 10.1063/1.3455643

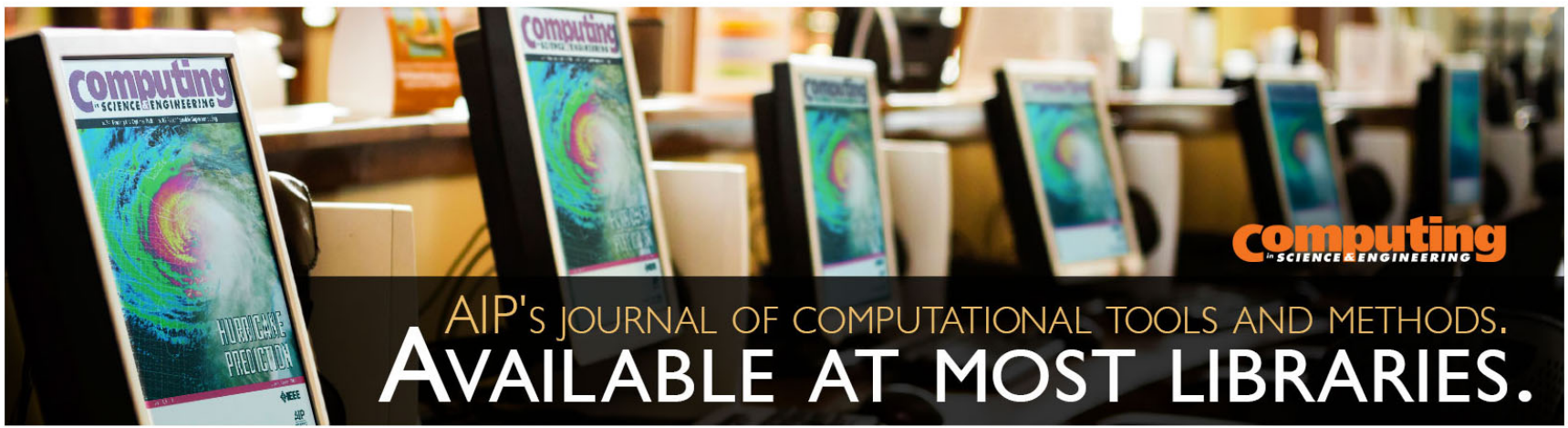




\title{
Stable planar single-layer hexagonal silicene under tensile strain and its anomalous Poisson's ratio
}

\author{
Baolin Wang, ${ }^{1}$ Jiangtao Wu, ${ }^{1}$ Xiaokun Gu, ${ }^{2}$ Hanqing Yin, ${ }^{1}$ Yujie Wei, ${ }^{1, a)}$ Ronggui Yang,, a) \\ and Mildred Dresselhaus ${ }^{3}$ \\ ${ }^{1}$ LNM, Institute of Mechanics, Chinese Academy of Sciences, Beijing 100190, People's Republic of China \\ ${ }^{2}$ Department of Mechanical Engineering, University of Colorado, Boulder, Colorado 80309, USA \\ ${ }^{3}$ Departments of Physics and Department of Electrical Engineering and Computer Sciences, Massachusetts \\ Institute of Technology, Cambridge, Massachusetts 02139, USA
}

(Received 17 November 2013; accepted 7 February 2014; published online 24 February 2014)

\begin{abstract}
Here, we report the structural and mechanical properties of several two-dimensional (2-D) materials by using first-principles density functional theory calculations. We find that the buckled single-layer silicene could transit to planar hexagonal silicene at a critical tensile strain of 0.20. Phonon dispersion analysis suggests that the planar hexagonal silicene under tension is stable. The Poisson's ratio of silicene and $\mathrm{MoS}_{2}$ shows strong anisotropy: it increases while stretched in the zigzag direction, but decreases when strained in the armchair direction. When stretched in the zigzag direction, the Poisson's ratio of silicene could reach 0.62. (C) 2014 AIP Publishing LLC.

[http://dx.doi.org/10.1063/1.4866415]
\end{abstract}

With the growing interest in the integration of 2-D materials, ${ }^{1-3}$ such as graphene and $\mathrm{MoS}_{2}$, with extraordinary properties for electronic applications, researchers find that the mechanical properties of such 2-D materials and their heterostructures are critical for their technical applications. $^{4-6}$ Many typical single-layer 2-D materials, for example, graphene, hexagonal boron-nitride (h-BN), fluorographene, $\mathrm{MoS}_{2}$, and silicene have been widely investigated. Although these 2-D materials all have a honeycomb arrangement, their properties can be very different. For example, $\mathrm{h}-\mathrm{BN}$ is electrical insulating with a large band gap both within and across the layers, in contrast to graphene with no band gap and being a semi-metal. Fluorographene is a derivative of graphene and can serve as an insulation layer for graphene electronics with a band gap of about $3 \mathrm{eV}^{7}$ Single layer $\mathrm{MoS}_{2}$ is a direct band gap semiconductor with a band gap of $1.8 \mathrm{eV} .{ }^{8}$ The interest in silicene stems from the great success in silicon technology. As a corollary, we desire to know whether the great success in silicon is reproducible in its 2-D counterpart. Unlike graphene, silicene has a hexagonal atomic arrangement and a buckled configuration. ${ }^{9}$ Such structure difference calls for an in-depth investigation on whether silicene exhibits different mechanical behavior in contract to graphene. The latter has been broadly recognized for its super-high strength ${ }^{4}$ and is regarded to have potential impact for composite materials. ${ }^{10,11}$ In this work, we perform close examination on the mechanical response of silicene by using first-principles density functional theory (DFT). For comparison, four typical other single layer 2-D materials including graphene, h-BN, fluorographene, and $\mathrm{MoS}_{2}$ are also investigated.

First-principles DFT calculations are performed to study the structural and mechanical properties of the five typical 2-D materials by using the Vienna $A b$ initio Simulation

\footnotetext{
a) Authors to whom correspondence should be addressed. Electronic addresses: yujie_wei@lnm.imech.ac.cn, Tel.: (86-10) 8254-4169 and ronggui.yang@colorado.edu, Tel.: (303)735-1003.
}

Package (VASP). ${ }^{12}$ The projector augmented wave (PAW) pseudopotentials ${ }^{13}$ and the generalized gradient approximation (GGA) of the Perdew-Burke-Ernzerhof (PBE) functional $^{14}$ are used. The kinetic-energy cut-off for the plane-wave basis set for silicene is $380 \mathrm{eV}$, and for graphene, $\mathrm{h}-\mathrm{BN}$, fluorographene and $\mathrm{MoS}_{2}$ the cutoff is $400 \mathrm{eV}$. For relaxation of the structures, $31 \times 31 \times 1$ Monkhorst-Pack $\mathrm{k}$-point mesh for graphene, h-BN and silicene, $16 \times 16 \times 1$ mesh for fluorographene and $5 \times 3 \times 1$ mesh for $\mathrm{MoS}_{2}$ are used. To eliminate the interactions between periodic images of single layer samples, a vacuum space of $20 \AA$ was used. Periodic boundary conditions are applied to the in-plane directions in all the calculations conducted here. Here, we make the assumption that the periodic unit cell with several atoms may represent well the mechanical response of large area perfect samples. All structures (graphene, h-BN, fluorographene, $\mathrm{MoS}_{2}$, and silicene) are relaxed using a conjugate gradient ( $\mathrm{CG}$ ) algorithm until the atomic forces are converged to $0.01 \mathrm{eV} / \AA$. The quasi-static displacement-controlled deformation is used by imposing the deformation with a small strain increment (a typical value of 0.01 is used for most calculations). The corresponding stress is obtained from the reaction force induced by the imposed strain divided by the current cross-sectional area (the current sample width times the sample thickness shown in Table I). So it is convenient to covert the stress-strain curves to force (per unit length) versus strain curves if the stress is multiplied by the sample thickness given in Table I. Phonon dispersion is analyzed by using the density functional perturbation theory $(\mathrm{DFPT})^{15}$ implemented in the Quantum Espresso (QE) Package. ${ }^{16}$ We used the pseudopotentials Si.pz-hgh.UPF, S.pbe-mt_fhi.UPF, and Mo.pbe-mt_fhi.UPF from QE website. The kinetic energy cutoff was set to be $45 \mathrm{Ry}$ for silicene and 70 Ry for $\mathrm{MoS}_{2}$.

Fig. 1(a) shows the stress versus strain curves of silicene in the armchair direction and in the zigzag direction. The mechanical behavior of silicene in the zigzag direction is substantially different from that in the armchair direction. 
TABLE I. The lattice parameters (from DFT calculation in this study and experiments if available) and the initial size of the simulation structure (Lx0 and Ly0) used in our DFT calculations for the five types of 2-D materials. Here, $a$ is the length of an in-plane bond and $c$ is the distance between two neighboring layers.

\begin{tabular}{lccccr}
\hline \hline Name & $a(\mathrm{~nm})(\mathrm{DFT})$ & $a(\mathrm{~nm})($ Exp. $)$ & $c(\mathrm{~nm})(\mathrm{DFT})$ & $c(\mathrm{~nm})($ Exp.) & $L_{x 0}$ \\
\hline Graphene & 0.247 & $0.245^{\mathrm{a}}$ & 0.33 & $0.33^{\mathrm{b}}$ & 0.2468 \\
h-BN & 0.250 & $0.249^{\mathrm{c}}$ & 0.33 & $0.33^{\mathrm{d}}$ & 0.4271 \\
Fluorographene & 0.254 & $0.248^{\mathrm{e}}$ & $0.62^{\mathrm{f}}$ & $0.62-0.87^{\mathrm{g}}$ & 0.2504 \\
MoS $_{2}$ & 0.318 & $0.315^{\mathrm{h}}$ & 0.614 & $0.675^{\mathrm{i}}$ & 0.4337 \\
Planar h-Si & 0.3901 & $\mathrm{~N} / \mathrm{A}$ & 0.42 & 0.6364 \\
Buckled h-Si & 0.3867 & $0.38^{\mathrm{j}}$ & $0.42^{\mathrm{k}}$ & 0.4415 \\
\hline
\end{tabular}

${ }^{\mathrm{a}}$ Reference 17 .

${ }^{\mathrm{b}}$ Reference 18.

${ }^{\mathrm{c}}$ Reference 19.

${ }^{\mathrm{d}}$ Reference 20 .

${ }^{\mathrm{e}}$ Reference 21.

${ }^{\mathrm{f}}$ Reference 22 .

${ }^{\mathrm{g}}$ Reference 23 .

${ }^{\text {h}}$ Reference 24.

${ }^{\mathrm{i}}$ Reference 25 .

${ }^{\mathrm{j}}$ Reference 26.

${ }^{\mathrm{k}}$ Reference 27.

There exist two stress peaks in the stress-strain curves when stretched in the zigzag direction. The first one corresponds to the mechanical response of buckled hexagonal silicene (buckled h-Si) structure. After the buckled sample is completely flattened by tension, a small stress drop emerges, corresponding to point "d" keyed in Fig. 1(a) (the amplified
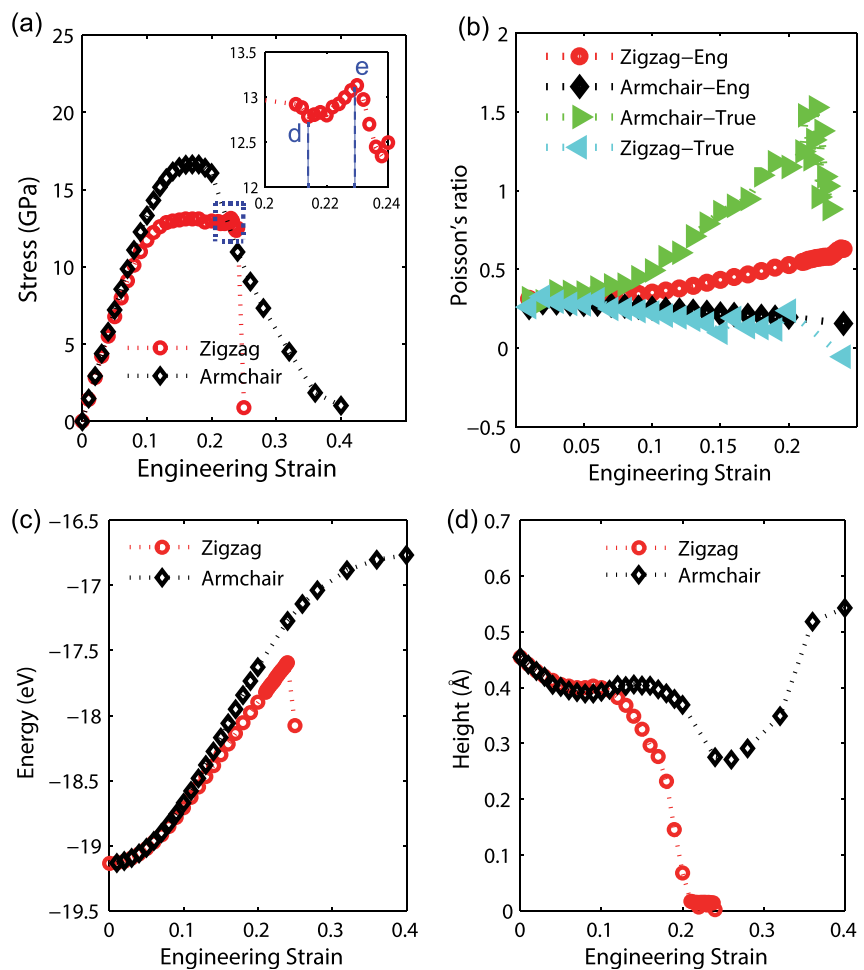

FIG. 1. Mechanical behavior of single layer silicene under tension. (a) The stress as a function of strain until the failure strain in both the armchair and the zigzag directions. The inset shows a small stress peak before the transition from buckled silicene to planar silicene. (b) The true and the engineering Poisson's ratio versus strains. The engineering Poisson's ratio can reach a large value of 0.62 when strained in the zigzag direction, which breaks the conventional upper bound of 0.5 . (c) Energy per atom versus strains. (d) Evolution of sample height to show the sudden flattening and structure transition while stretching along the zigzag direction. region in the inset). Further stretching the planar hexagonal silicene (planar h-Si) may induce a transition to square lattice (s-Si), which however, is not a stable structure even under straining, as will be detailed in Fig. 2.

When strain is applied in the longitudinal direction, the samples are relaxed to ensure that the load in the transverse direction approaches zero. The degree of deformation in the transverse direction of the sample over that along the longitudinal direction is characterized by the Poisson's ratio. It is well-known that the Poisson's ratio is bounded in the range of $(-1.0,0.5)$ in isotropic materials. ${ }^{28,29}$ Materials with $\nu>0.5$
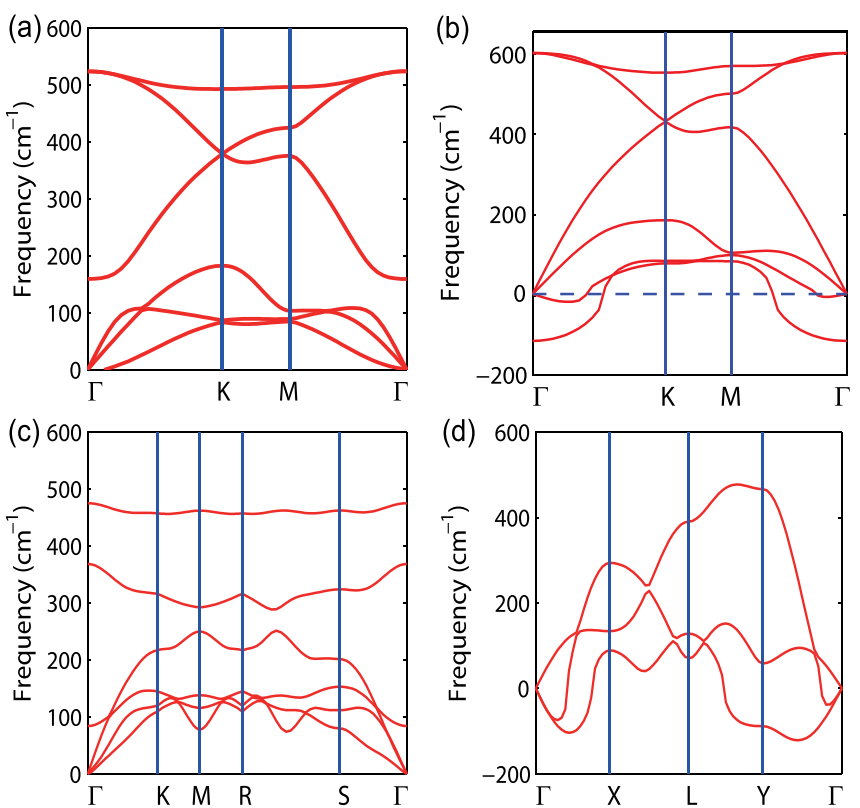

FIG. 2. Phonon dispersion of silicene at different configurations and strains. (a) Initially buckled configuration. (b) Silicene with planar honeycomb structure without straining, suggesting unstable configuration without straining. (c) At $20 \%$ strain for buckled silicene and now the sample is planar, suggesting stable configuration of planar silicene with straining. (d) At $36 \%$ strain for buckled silicene, now we have square silicene, where phonon instability is seen. 
will counter-intuitively undergo "stretch densifying," ${ }^{30}$ which might find various applications. ${ }^{30}$ Following the original definition for the Poisson's ratio $\nu$, we have

$$
\nu=-\varepsilon_{\mathrm{y}} / \varepsilon_{\mathrm{x}}=-\left(L_{\mathrm{y}}-L_{\mathrm{y} 0}\right) L_{\mathrm{x} 0} / L_{\mathrm{y} 0}\left(L_{\mathrm{x}}-L_{\mathrm{x} 0}\right),
$$

where $\varepsilon_{\mathrm{x}}$ and $\varepsilon_{\mathrm{y}}$ are the engineering strains in the loading and the transverse directions, respectively, $L_{\mathrm{x} 0}$ and $L_{\mathrm{y} 0}$ are the initial lengths of the sample in the loading (longitudinal) direction and in the transverse direction, respectively, and $L_{\mathrm{x}}$ and $L_{\mathrm{y}}$ are the respective deformed dimensions. Following mechanical convention, the Poisson's ratio defined above is called as the engineering Poisson's ratio. For comparison, we also give the true Poisson's ratio by calculating $\nu$ at the deformed configuration, i.e.,

$$
\nu=-\left(L_{\mathrm{y}}(t+d t)-L_{\mathrm{y}}(t)\right) L_{\mathrm{x}}(t) / L_{\mathrm{y}}(t)\left(L_{\mathrm{x}}(t+d t)-L_{\mathrm{x}}(t)\right),
$$

where the dimensions at time $t$ and subsequent time step $t+d t$ are used. From both the true and the engineering Poisson's ratio versus strain curves shown in Fig. 1(b), we see that although $\nu$ keeps decreasing with increasing strain in the armchair direction, it increases with straining in the zigzag direction. The engineering Poisson's ratio of silicene indeed breaks the conventional upper bound at strains from 0.19 to 0.24 and is anomalously greater than 0.5 . Figure 1 (c) shows the height of the buckled layer as a function of strains in both the armchair and zigzag directions, which demonstrates the transition from buckled b-Si to planar h-Si. The energy as a function of strains (Fig. 1(d)) also shows an apparent kink at the point where the structure changes. We also find that the buckled h-Si state is most stable when the strain is less than 0.20 , which is consistent with experimental observations. ${ }^{9}$ The structure becomes the planar h-Si structure in the strain range of $0.20-0.24$.

While the structure transition seen in Fig. 1-from both buckled $\mathrm{h}-\mathrm{Si}$ to planar $\mathrm{h}$-Si-seems intriguing, it remains unclear whether such intermediate configuration is stable. We use the DFPT to perform phonon calculations. ${ }^{15}$ The obtained phonon dispersion could be used to determine the stability of 2-D structures, with negative frequencies suggesting structural instability. ${ }^{31}$ Figure 2 (a) shows the phonon dispersions of buckled silicene at $0 \%$ strain; as a comparison, that for planar silicene without straining is given in Fig. 2(b). The negative frequency for the planar silicene without straining suggests the instability of this structure, which is consistent with previous analysis. ${ }^{32}$ Figure 2 (c) gives the phonon dispersions of the buckled silicene at $20 \%$ tensile strain, where it becomes planar. At $20 \%$ strain, the structure is still stable. When further straining is applied, we see emergence of negative frequency for buckled silicene at $36 \%$ (now the structure changes to square silicene, and one-atom unit cell was used to calculate the phonon dispersion). Those phonon dispersion curves shown in Fig. 2 indicate that silicene is structurally stable even during the stress softening region while we apply strain to the buckled silicene.

As a comparison, we also explored the stress-strain response of $\mathrm{MoS}_{2}$. Figure 3(a) shows evolutions of both the Poisson's ratio and the stress as a function of strain in single

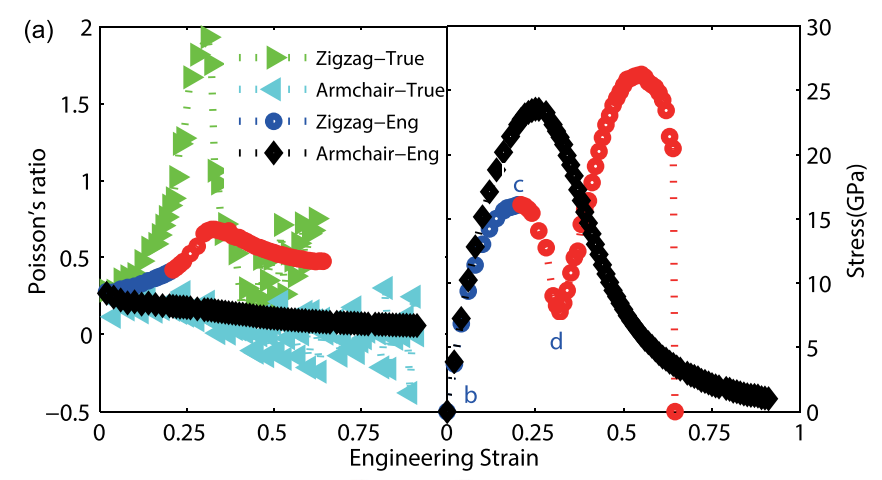

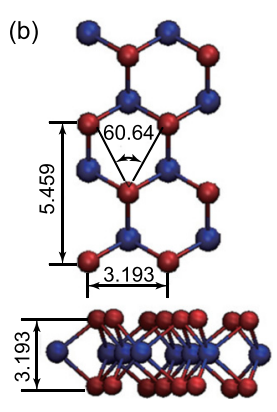

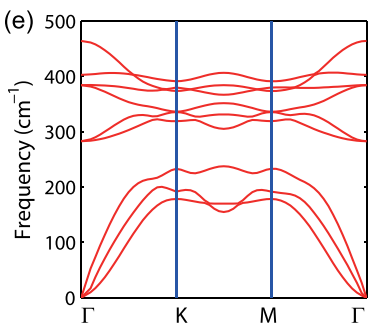

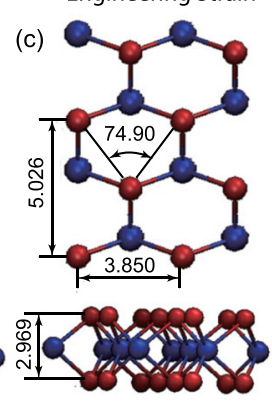
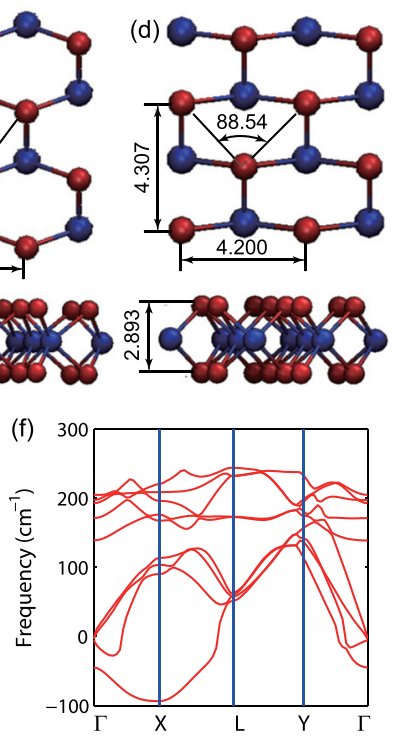

FIG. 3. Mechanical behavior of $\mathrm{MoS}_{2}$ and its phonon dispersion at different strains. (a) The true and the engineering Poisson's ratio (left) and stress (right) as a function of strain in both the armchair and the zigzag directions. Note that the place where the Poisson's ratio beyond the peak point "c" is in red. (b)-(d) Deformed atomic structures at different stage of strains keyed in the stress-strain curve in (a). (e) and (f) Phonon dispersion of $\mathrm{MoS}_{2}$ at its initial configuration and at $40 \%$ strain (with 6 atoms in orthogonal super cell), respectively.

layer $\mathrm{MoS}_{2}$ stretched in both the armchair direction and the zigzag direction. The Poisson's ratio in $\mathrm{MoS}_{2}$ increases with the applied strain when stretched in the zigzag direction, but decreases when the strain is applied in the armchair direction. Figures 3(b)-3(d) show in turn the bond configurations (both in plane and transverse projections) at different stage of deformation. By comparing both the stress-strain curve and the bond configurations, we see that the first peak occurs at a strain of 0.22 corresponding to the maximum stress achievable in the hexagonal-close packed (hcp) $\mathrm{MoS}_{2}$, but the second one at the strain of 0.55 may be due to the transition of hep $\mathrm{MoS}_{2}$ to body-centered tetragonal (bct) $\mathrm{MoS}_{2}$. Results of phonon dispersion analysis shown in Figs. 3(e) and 3(f), at the strain of 0.0 and 0.4 , suggest that the (bct) $\mathrm{MoS}_{2}$ is not stable. For completeness, we further explore the mechanical behaviors of three other 2-D materials, graphene, h-BN, and fluorographene. The results are shown in Fig. 4. The mechanical responses of these three materials are strikingly different from that of $\mathrm{MoS}_{2}$ and silicene. The Poisson's ratio decreases monotonically with strains in graphene, h-BN, and fluorographene when stretched in the zigzag direction.

In summary, we report the structural and mechanical properties of several 2-D materials by using first-principles 

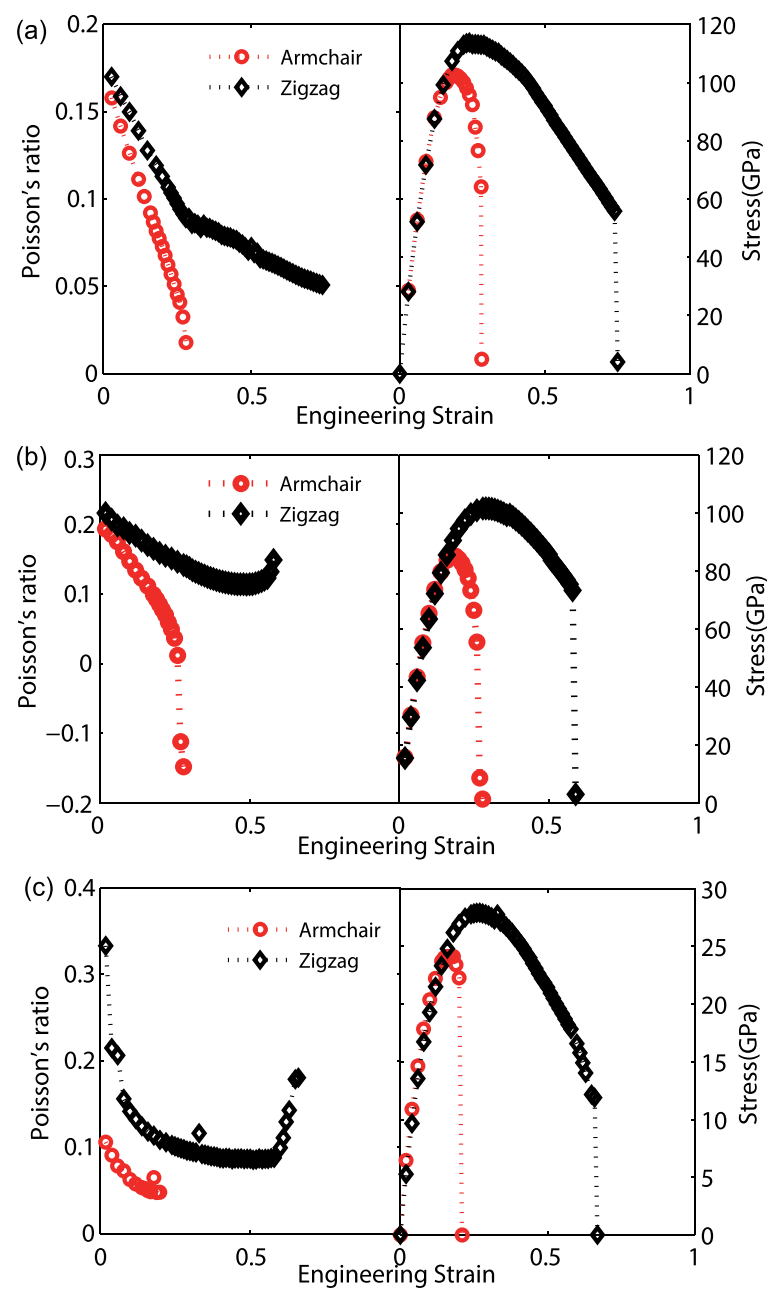

FIG. 4. The engineering Poisson's ratio (left) and stress (right) as a function of strain until the failure strain in both the armchair and the zigzag directions in: (a) Graphene; (b) h-BN; and (c) Fluorographene.

DFT calculations. We show an abrupt structure transition in buckled planar silicene to planar silicene at a critical tensile strain of 0.20 . Phonon dispersion analysis suggests that the planar hexagonal silicene under tension is stable, and silicene can be structurally stable even during the stress softening region. The Poisson's ratio of silicene shows strong anisotropy: it increases while stretched in the zigzag direction and could reach 0.62 , a number breaking the conventional boundary of Poisson's ratio of three-dimensional materials, but the Poisson's ratio decreases when strained in the armchair direction. In contrast, the Poisson's ratio decreases with strains in graphene, h-BN, and fluorographene when stretched in the zigzag direction. The stability of planar silicene suggests that silicene is intrinsically tougher than other 2-D materials as their out-of-plane bonds are both bendable and extensible to become in-plane bonds. Such mechanism could be utilized in making composites based on 2-D materials. ${ }^{10,11}$ It also shows possibility that we may synthesize planar silicene when the lattice mismatch between the substrate material and planar silicene is within the right range, so that the interfacial energy between the substrate and the silicene is able to overcome the strain energy required to flatten buckled silicene. Furthermore, the distinct Poisson's ratio effect observed in those 2-Dimensional may require us to pay extra attention while designing heterostructures where mechanical deformation presents: The different Poisson's effect in different layers could induce significant shearing between them.

Y.W. acknowledges the support from Chinese Academy of Sciences (CAS), National Natural Science Foundation of China (NSFC) (Nos. 11021262 and 11272327), and MOST 973 of China (No. 2012CB937500). Computation is supported by the Computation Center of CAS. R.Y. acknowledges the support from NSF and AFOSR. M.S.D. acknowledges the support of NSF/DMR-10-04147.

${ }^{1}$ L. Britnell, R. V. Gorbachev, R. Jalil, B. D. Belle, F. Schedin, A. Mishchenko, T. Georgiou, M. I. Katsnelson, L. Eaves, S. V. Morozov, N. M. R. Peres, J. Leist, A. K. Geim, K. S. Novoselov, and L. A. Ponomarenko, Science 335, 947 (2012).

${ }^{2}$ M. P. Levendorf, C. J. Kim, L. Brown, P. Y. Huang, R. W. Havener, D. A. Muller, and J. Park, Nature 488, 627 (2012).

${ }^{3}$ H. Yang, J. Heo, S. Park, H. J. Song, D. H. Seo, K.-E. Byun, P. Kim, H.-J. Yoo, and K. Kim, Science 336, 1140 (2012).

${ }^{4}$ C. Lee, X. Wei, J. W. Kysar, and J. Hone, Science 321, 385 (2008).

${ }^{5}$ S. P. Koenig, N. G. Boddeti, M. L. Dunn, and J. S. Bunch, Nat. Nanotechnol. 6, 543 (2011).

${ }^{6}$ N. N. Klimov, S. Jung, S. Zhu, T. Li, C. A. Wright, S. D. Solares, D. B. Newell, N. B. Zhitenev, and J. A. Stroscio, Science 336, 1557 (2012).

${ }^{7}$ R. R. Nair, W. C. Ren, R. Jalil, I. Riaz, V. G. Kravets, L. Britnell, P. Blake, F. Schedin, A. S. Mayorov, S. Yuan, M. I. Katsnelson, H. M. Cheng, W. Strupinski, L. G. Bulusheva, A. V. Okotrub, I. V. Grigorieva, A. N. Grigorenko, K. S. Novoselov, and A. K. Geim, Small 6, 2877 (2010).

${ }^{8}$ K. F. Mak, C. Lee, J. Hone, J. Shan, and T. F. Heinz, Phys. Rev. Lett. 105, 136805 (2010).

${ }^{9}$ B. Lalmi, H. Oughaddou, H. Enriquez, A. Kara, S. Vizzini, B. Ealet, and B. Aufray, Appl. Phys. Lett. 97, 223109 (2010).

${ }^{10}$ S. Stankovich, D. A. Dikin, G. H. B. Dommett, K. M. Kohlhaas, E. J. Zimney, E. A. Stach, R. D. Piner, S. T. Nguyen, and R. S. Ruoff, Nature 442, 282 (2006).

${ }^{11}$ T. Ramanathan, A. A. Abdala, S. Stankovich, D. A. Dikin, M. HerreraAlonso, R. D. Piner, D. H. Adamson, H. C. Schniepp, X. Chen, R. S. Ruoff, S. T. Nguyen, I. A. Aksay, R. K. Prud'Homme, and L. C. Brinson, Nat. Nanotechnol. 3, 327 (2008).

${ }^{12}$ G. Kresse and J. Furthmüller, Comput. Mater. Sci. 6, 15 (1996); Phys. Rev. B 54, 11169 (1996).

${ }^{13}$ P. E. Blöchl, Phys. Rev. B 50, 17953 (1994); G. Kresse and D. Joubert, ibid. 59, 1758 (1999).

${ }^{14}$ J. P. Perdew, K. Burke, and M. Ernzerhof, Phys. Rev. Lett. 77, 3865 (1996); 78, 1396 (1997).

${ }^{15}$ S. Baroni, S. de Gironcoli, A. Dal Corso, and P. Giannozzi, Rev. Mod. Phys. 73, 515 (2001).

${ }^{16}$ P. Giannozzi, S. Baroni, N. Bonini, M. Calandra, R. Car, C. Cavazzoni, D. Ceresoli, G. L. Chiarotti, M. Cococcioni, I. Dabo, A. Dal Corso, S. de Gironcoli, S. Fabris, G. Fratesi, R. Gebauer, U. Gerstmann, C. Gougoussis, A. Kokalj, M. Lazzeri, L. Martin-Samos, N. Marzari, F. Mauri, R. Mazzarello, S. Paolini, A. Pasquarello, L. Paulatto, C. Sbraccia, S. Scandolo, G. Sclauzero, A. P. Seitsonen, A. Smogunov, P. Umari, and R. M. Wentzcovitch, J. Phys.: Condens. Matter 21, 395502 (2009).

${ }^{17}$ A. T. N. Diaye, J. Coraux, T. N. Plasa, C. Busse, and T. Michely, New J. Phys. 10, 043033 (2008).

${ }^{18}$ Y. Baskin and L. Meyer, Phys. Rev. 100, 544 (1955).

${ }^{19}$ N. Alem, R. Erni, C. Kisielowski, M. Rossell, W. Gannett, and A. Zettl, Phys. Rev. B 80, 155425 (2009).

${ }^{20}$ Y. Shi, C. Hamsen, X. Jia, K. K. Kim, A. Reina, M. Hofmann, A. L. Hsu, K. Zhang, H. Li, Z.-Y. Juang, M. S. Dresselhaus, L.-J. Li, and J. Kong, Nano Lett. 10, 4134 (2010).

${ }^{21}$ K. S. Novoselov, A. K. Geim, S. V. Morozov, D. Jiang, Y. Zhang, S. V. Dubonos, I. V. Grigorieva, and A. A. Firsov, Science 306, 666 (2004).

${ }^{22}$ S. Helveg, J. V. Lauritsen, E. Lægsgaard, I. Stensgaard, J. K. Nørskov, B. S. Clausen, H. Topsøe, and F. Besenbacher, Phys. Rev. Lett. 84, 951 (2000).

${ }^{23}$ M. M. Benameur, B. Radisavljevic, J. S. Héron, S. Sahoo, H. Berger, and A. Kis, Nanotechnology 22, 125706 (2011).

${ }^{24}$ S. S. Han, T. H. Yu, B. V. Merinov, A. C. T. Van Duin, R. Yazami, and W. A. Goddard III, Chem. Mater. 22, 2142 (2010). 
${ }^{25}$ K. Zboril, F. Karlicky, A. B. Bourlinos, T. A. Steriotis, A. K. Stubos, V. Georgakilas, K. Safarova, D. Jancík, C. Trapalis, and M. Otyepka, Small 6, 2885 (2010).

${ }^{26}$ L. Chen, C. C. Liu, B. Feng, X. He, P. Cheng, Z. Ding, S. Meng, Y. Yao, and K. Wu, Phys. Rev. Lett. 109, 056804 (2012).

${ }^{27}$ M. Hu, X. Zhang, and D. Poulikalos, Phys. Rev. B 87, 195417 (2013).

${ }^{28}$ G. N. Greaves, A. L. Greer, R. S. Lakes, and T. Rouxel, Nature Mater. 10, 823 (2011).
${ }^{29}$ Y. C. Fung, Foundation of Solid Mechanics (Prentice-Hall, Englewood Cliffs, New Jersey, 1968).

${ }^{30}$ R. H. Baughman and D. S. Galvao, Nature 365, 735 (1993); R. H. Baughman, S. O. Dantas, S. Stafström, A. A. Zakhidov, T. B. Mitchell, and D. H. E. Dubin, Science 288, 2018 (2000).

${ }^{31}$ F. Liu, P. Ming, and J. Li, Phys. Rev. B 76, 064120 (2007).

${ }^{32}$ S. Cahangirov, M. Topsakal, E. Akturk, H. Sahin, and S. Ciraci, Phys. Rev. Lett. 102, 236804 (2009). 